Palavras chave:

Branqueamento

Classificação de fibras

Refino

Histórico:

Recebido I / 04/2012

Aceito 16/10/2013

Keywords:

Bleaching

Fiber fractionating

Refining

Correspondência: jaquecomelato@hotmail.com
Jaqueline Silveira Comelato Favaro', Gustavo Ventorim', José Cláudio Caraschi'

\section{EFEITO DE REAGENTES DE BRANQUEAMENTO NA CLASSIFICAÇÃO E REFINAÇÃO DE POLPA DE EUCALIPTO}

RESUMO: Objetivou-se, neste estudo, avaliar o comportamento do branqueamento de polpa de celulose kraft de eucalipto nas propriedades físicas e mecânicas do papel. A polpa foi branqueada por quatro sequências semelhantes, sendo elas: $D(E+P) D D$ (referência), $A_{H T} D(E+P) D D, D(E+P) D(P O), P_{X}(E+P) D D$. Cada uma das sequências contendo um dos reagentes analisados: dióxido de cloro, ácido sulfúrico peróxido de hidrogênio pressurizado, e perácido (ácido de Caro). Parte das amostras foi refinada para $40^{\circ} \mathrm{SR}$, enquanto a outra parte foi classificada em equipamento Bauer-McNett. Posteriormente, a porção retida na malha de 50 mesh, foi refinada ao mesmo número de revoluções que sua amostra global. As polpas branqueadas com $A_{H T} P_{X}$ e $P O$ apresentaram diminuição da resistência mecânica e aumentaram a concentração de polpa em peneiras mais finas, durante a classificação, pois esses reagentes são pouco seletivos. Os resultados encontrados mostraram que polpas branqueadas por reagentes como o perácido e peróxido de hidrogênio pressurizado indicaram menores resistências, assim como suas frações de polpa. No entanto, apesar da baixa seletividade da hidrólise ácida, foi verificado que essa sequência apresentou altos índices de rasgo e tração, tanto da polpa global quanto da polpa classificada, concluindo que as polpas apesar de classificadas, apresentam elevada resistência mecânica.

\section{EFFECT OF BLEACHING REAGENTS ON EUCALYPTUS KRAFT PULP FRACTIONATION AND REFINING}

ABSTRACT: The aim of this study was to evaluate the behavior of eucalyptus kraft pulp bleaching on paper physical and mechanical properties. Pulp was bleached by four similar sequences, each containing one of the reagents analyzed: $D(E+P) D D$ (reference), $A_{H T} D(E+P) D D, D(E+P) D(P O)$, and $P_{X}(E+P) D D$. Each of the sequences contained one of the studied reagents: chlorine dioxide, sulfuric acid, pressurized hydrogen peroxide, and peracid (Caro's acid). One part of the samples was refined to $40^{\circ} \mathrm{SR}$, whereas the other part was classified in a Bauer-McNett. Subsequently, the part retained in 50 mesh sieve, was refined to the same number of revolutions as the global sample. Pulp bleached in $A_{H T}, P O$, and $P_{X}$ exhibited reduced mechanical strength and during fractionating increased the concentration of fibers in finer sieves because these reagents are less selective. The results show that pulp bleached in reagents such as pressurized hydrogen peroxide and peracid indicated reduced strength and produced greater fiber concentrations. However, despite the low selectivity of acid hydrolysis, it was found that this sequence had very high tear and tensile strength comparable to classified and global pulp, concluding that regardless of classification, pulp fractions may have high mechanical strength.
DOI:
' Universidade Estadual Paulista Júlio de Mesquita Filho - Itapeva, São Paulo, Brasil 


\section{INTRODUÇÃO}

O Brasil se destaca por possuir tecnologia bem estabelecida no branqueamento de fibra curta, especialmente de eucalipto, sendo o quarto maior produtor mundial (ASSOCIAÇÃO BRASILEIRA DE CELULOSE E PAPEL - BRACELPA, 20I0) e um dos três países mais competitivos do mercado internacional de celulose (CARVALHO et al., 2009).

Por ser facilmente branqueado, o eucalipto é muito utilizado para a produção de papéis de alta alvura. Esse branqueamento tende a reduzir o potencial de resistência da fibra, de modo que cada reagente utilizado afeta diferentemente as propriedades da celulose produzida. Isso ocorre em razão, principalmente, do uso de reagentes como oxigênio e seus derivados (ozônio, peróxido de hidrogênio e perácidos) os quais são menos seletivos para a preservação do potencial de resistência. $\mathrm{O}$ uso de diferentes técnicas de polpação e branqueamento altera a química das macromoléculas nas fibras da madeira, os quais afetam as propriedades de inchamento e absorção de água pela fibra (ANDRADE; COLODETTE, 2010) alterando, consequentemente, as propriedades dos papéis produzidos.

A quantidade de polpa de madeiras de fibra curta utilizada nos dias de hoje em vários tipos de papeis poderia ser melhorada e novos usos poderiam ser criados se as propriedades de resistência forem melhoradas, sem sacrificar outras propriedades desejáveis. A qualidade das fibras, as quais estão relacionadas com as suas propriedades morfológicas, são muito importantes para a qualidade da folha de papel. As fibras longas criam a malha principal sobre a qual as fibras curtas irão ser retidas e aglomeradas para a formação do papel (CORSON, 1989), sendo que a resistência do papel depende, consideravelmente, do comprimento da fibra.

$O$ fracionamento de fibras em classificadores, $O$ qual é capaz de separar fibras em grupos de acordo com seu tamanho, é um método de melhorar a qualidade do papel. As frações podem ter diferentes utilizações finais individualmente, ou ser recombinados após a classificação (SETH, 1999). Muitos pesquisadores estudaram a classificação de fibras em Bauer-McNett (CARVALHO et al., 1997; GOODING; OLSON, 200I; SILVA et al., 1997) e seus benefícios. Os resultados encontrados podem ser variados, já que as operações de polpação e branqueamento modificam a natureza dos constituintes químicos das fibras, vasos e finos celulósicos.

$\mathrm{Na}$ classificação de fibras, quando o tamanho da malha do classificador diminui, há maior presença de fibras quebradas, colapsadas e dobradas. Quanto mais se aproxima da fração de comprimentos de fibras menores, maiores as presenças de pedaços de fibras e paredes de fibras deformadas (CARVALHO et al., 1997; SETH, 1999).

O refino da celulose é um procedimento que visa a elevar a resistência física do papel, devido à maior conformação e uniformidade das fibras (BANAVATH et al., 20II; TAIPALE et al., 20I0), no entanto, reduz as propriedades ópticas (LUNDIN et al., 2009). Ao atingirem um ponto máximo de refino, as propriedades da folha tendem a se estabilizar e, se o refino for prolongado, ele pode levar ao declínio (GIGAC; FIŠEROVÁ, 2008; LUNDIN et al., 2009). O refino das frações de polpas classificadas permite atribuir melhor resistência a um determinado grupo de fibra. Acarreta em modificações morfológicas das fibras, aumenta a concentração de fibras menores, pela deterioração das fibras longas, aumenta a concentração de finos e melhora a formação da folha, já que esses desempenham $\circ$ papel de elementos ligantes, preenchendo os espaços vazios entre as fibras (CORSON, 1989; VOMHOFF; GRUNDSTRÖM, 2003; ZAYTSEVA, 20I0).

Visando a avaliar os impactos de reagentes de branqueamento na qualidade da polpa celulósica, neste trabalho, objetivou-se investigar o comportamento do branqueamento de celulose kraft de eucalipto sobre as fibras classificadas e não classificadas, por meio de diferentes sequências e sua influência sobre as propriedades físicas e mecânicas do papel.

\section{MATERIAL E MÉTODOS}

Uma amostra de polpa celulósica industrial de eucalipto pré-deslignificada com oxigênio foi utilizada neste estudo. As características iniciais da polpa foram: número kappa de 9,8 , alvura de $60,2 \%$ ISO e viscosidade de 28,3 cP. Neste estudo, realizaram-se quatro sequências de branqueamento pelo processo ECF, sendo elas: $D(E+P) D D$ (referência), $A_{H T} D(E+P) D D, D(E+P)$ $D(P O), P_{X}(E+P) D D$, cujas condições são apresentadas na Tabela I.

O branqueamento com os estágios não pressurizados foram efetuados em sacos de polietileno com amostras representativas de polpa. O licor de branqueamento foi adicionado à polpa em temperatura ambiente. Terminada a reação, foram extraídas amostras de licor residual das polpas para análises de $\mathrm{pH}$ e residual (quando aplicável). A polpa foi lavada com o equivalente a $9 \mathrm{~m}^{3}$ de água por tonelada de polpa absolutamente seca. 
TABELA I Condições gerais de branqueamento.

TABLE I General bleaching conditions.

\begin{tabular}{|c|c|c|c|c|c|c|c|}
\hline \multirow{2}{*}{ Condições } & \multicolumn{7}{|c|}{ Estágios de branqueamento } \\
\hline & $\mathrm{D}_{0}$ & $\mathrm{~A}_{\mathrm{HT}}$ & $P_{x}$ & $(E+P)$ & $D_{1}$ & $\mathrm{D}_{2}$ & $\mathrm{PO}$ \\
\hline Consistência (\%) & 10 & 10 & 10 & 10 & 10 & 10 & 10 \\
\hline Tempo (min) & 30 & 120 & 30 & 60 & 180 & 180 & 60 \\
\hline Temperatura $\left({ }^{\circ} \mathrm{C}\right)$ & 60 & 95 & 60 & 70 & 70 & 70 & 105 \\
\hline Pressão (kPa) & - & - & - & - & - & - & 500 \\
\hline $\mathrm{pH}$ final & 2,7 & 2,0 & 3,0 & 11,2 & 3,8 & 4,1 & 10,5 \\
\hline $\mathrm{NaOH}, \mathrm{kg} \cdot \mathrm{t}^{-1}$ & - & - & **⿻丷木 & 8,0 & - & **⿻丷木 & 8,0 \\
\hline $\mathrm{H}_{2} \mathrm{SO}_{4}, \mathrm{~kg} \cdot \mathrm{t}^{-1}$ & **⿻丷木 & *⿻丷木 & & - & *** & - & - \\
\hline $\mathrm{O}_{2}, \%$ & - & - & - & - & - & - & 2,0 \\
\hline $\mathrm{ClO}_{2}, \mathrm{~kg} \cdot \mathrm{t}^{-1}$ como $\mathrm{Cl}_{2}$ & $\mathrm{FK}^{\mathrm{I}}=0,2$ & - & - & - & * & * & - \\
\hline $\mathrm{H}_{2} \mathrm{O}_{2}, \mathrm{~kg} \cdot \mathrm{t}^{-1}$ & - & - & - & 3,0 & - & - & 3,0 \\
\hline$P_{X}, \mathrm{~kg} \cdot \mathrm{t}^{-1}$ & - & - & 5,0 & - & - & - & - \\
\hline
\end{tabular}

'Fator kappa. * Dosagem otimizada para obter alvura acima de 89,5\% ISO. ** Dosagem otimizada para ajuste de $\mathrm{pH}$.

O estágio com peróxido pressurizado (PO) foi efetuado, em um digestor com quatro reatores individuais de aço inox, com capacidade de I,5 litros cada, com ajuste de temperatura e pressão. Em cada cápsula foram colocadas $110 \mathrm{~g}$ a.s. de polpa. Foi adicionada à polpa uma mistura de água para ajuste da consistência, $\mathrm{NaOH}$ para ajuste do $\mathrm{pH}_{\text {e }} \mathrm{H}_{2} \mathrm{O}_{2}$ para o branqueamento. Após atingir a temperatura estabelecida, sob o efeito da rotação constante do digestor, foi aplicada a carga de oxigênio mantendo a pressão de $500 \mathrm{kPa}$. Ao término da deslignificação, a pressão do digestor foi aliviada para retirar as polpas das cápsulas. Foram extraídas amostras de licor residual das polpas para análise de $\mathrm{pH}$. Em seguida, a polpa foi lavada com o equivalente a $9 \mathrm{~m}^{3} \mathrm{de}$ água por tonelada de polpa absolutamente seca.

As análises de número kappa, alvura e viscosidade da polpa foram medidas pelos métodos TAPPI-236 om99, TAPPI-525 om92, TAPPI-230 om99, respectivamente. As folhas de polpas para a reversão de alvura foram submetidas em estufa, durante quatro horas, em nível de $0 \%$ de umidade relativa e a uma temperatura de $105^{\circ} \mathrm{C}$, sendo sua alvura medida em seguida TAPPI-525 om 92 (TECHNICAL ASSOCIATION OF THE PULP AND PAPER INDUSTRY - TAPPI, 2002).

O custo total do branqueamento foi calculado com base nas dosagens $\left(\mathrm{kg} \cdot \mathrm{t}^{-1}\right.$ a.s. $)$ de reagente aplicado em cada estágio e no preço (US\$. $\mathrm{kg}^{-1}$ ) dos reagentes utilizados. O custo total foi determinado pelo somatório dos custos de reagentes de cada estágio individual. $O$ preço dos reagentes (US $\$ / \mathrm{kg}$ do produto) foi usado para calcular os custos dos reagentes químicos (base 100\%) de branqueamento: dióxido de cloro $\left(\mathrm{ClO}_{2}\right)=1,0$; peróxido de hidrogênio $\left(\mathrm{H}_{2} \mathrm{O}_{2}\right)=0,85$; oxigênio $\left(\mathrm{O}_{2}\right)=$ 0 , I0; hidróxido de sódio $(\mathrm{NaOH})=0,50$; ácido sulfúrico $\left(\mathrm{H}_{2} \mathrm{SO}_{4}\right)=0,08$ e ácido de Caro $\left(\mathrm{H}_{2} \mathrm{SO}_{5}\right)=1,5$.

Parte das polpas foi classificada em equipamento Bauer-McNett de acordo com a norma TAPPI-233 cm95, com quatro repetições cada para a determinação da quantidade de polpa retida em cada tanque. Após essa determinação, foram conduzidas mais 20 repetições, sendo essas amostras armazenadas para refino, formação de folhas e análises posteriores.

Tanto a porção da polpa global (não classificada) como a porção classificada foram refinadas em moinho $\mathrm{PFI}$, em duplicata. Foi estabelecido que os testes físicos e mecânicos fossem realizados para as polpas globais com $40^{\circ}$ Schopper-Riegler ( ${ }^{\circ} \mathrm{SR}$ ). As polpas classificadas retidas na malha de 50 mesh do classificador foram refinadas ao mesmo número de revoluções que a sua amostra global. Os ensaios de resistência ao rasgo (TAPPI - 414 om 98), resistência à tração (TAPPI - 494 om 96), peso específico aparente e volume específico aparente (TAPPI - $220 \mathrm{sp}$ 96) e de ascensão capilar Klemm (TAPPI - 44I om-98) foram efetuados com 10 repetições cada.

As fibras das polpas branqueadas foram avaliadas utilizando-se analisador óptico automático FQA - Fiber Quality Analyser, modelo LDA02, do fabricante OpTest. Os parâmetros analisados foram comprimento médio ponderado por comprimento, a coarseness da fibra e o teor de finos. Essas determinações foram efetuadas em 16 amostras de polpas branqueadas, sendo quatro globais e quatro classificadas na malha de 50 mesh do classificador, com 2 repetições cada.

$\mathrm{Na}$ análise estatística dos resultados aplicou-se a análise de variância, por meio do teste $\mathrm{F}$ aos níveis de $\mathrm{I} \%$ e $5 \%$ de probabilidade. Utilizou-se também do teste de Tukey para a comparação entre médias dos tratamentos, ao nível de $5 \%$ de probabilidade.

\section{RESULTADOS E DISCUSSÃO}

\section{Branqueamento}

Os resultados do branqueamento das quatro amostras foram interpretados com base nos parâmetros de qualidade das polpas branqueadas, como os valores de alvura, reversão de alvura, viscosidade e custos de branqueamento, conforme descritos na Tabela 2 .

A reversão de alvura foi menor para a polpa com o estágio $\mathrm{A}_{\mathrm{HT}}$, apresentando a reversão de apenas I, I\% ISO, que pode ser explicado pela hidrólise ácida, 
TABELA 2 Características das polpas e custos dos branqueamentos.

TABLE 2 Pulp characteristics and bleaching costs.

\begin{tabular}{|c|c|c|c|c|}
\hline \multirow{2}{*}{ Resultados } & \multicolumn{4}{|c|}{ Sequências } \\
\hline & Ref. & $\mathrm{A}_{\mathrm{HT}}$ & $\mathrm{PO}$ & $P_{x}$ \\
\hline Alvura A. D.', \% ISO & 90,2 & 90,4 & 90,9 & 89,5 \\
\hline Reversão de Alvura O. D. ${ }^{2}, \%$ ISO & 2,9 & I,I & 1,3 & 1 \\
\hline Viscosidade, cP & 24,2 & 21,8 & 22,2 & 22 \\
\hline $\mathrm{ClO}_{2}$ como $\mathrm{Cl}_{2}, \mathrm{~kg} \cdot \mathrm{t}^{-1}$ & 35,6 & 30,6 & 32,6 & 29, \\
\hline $\mathrm{H}_{2} \mathrm{O}_{2}, \mathrm{~kg} \cdot \mathrm{t}^{-1}$ & 3,0 & 3,0 & 6,0 & 3, \\
\hline $\mathrm{NaOH}, \mathrm{kg} \cdot \mathrm{t}^{-1}$ & 7,8 & 8,0 & 16,8 & 33,0 \\
\hline $\mathrm{H}_{2} \mathrm{SO}_{4}, \mathrm{~kg} \cdot \mathrm{t}^{-1}$ & 1,0 & 5,0 & 1,0 & 0, \\
\hline$P_{X}, k g \cdot t^{-1}$ & 0,0 & 0,0 & 0,0 & $5,($ \\
\hline $\mathrm{O}_{2}, \mathrm{~kg} \cdot \mathrm{t}^{-1}$ & 0,0 & 0,0 & 20,0 & 0,0 \\
\hline Cloro ativo total, $\mathrm{kg} \cdot \operatorname{tas}^{-1}$ & 41,9 & 36,9 & 45,1 & 45,7 \\
\hline Custo Total de Reagentes, US\$.t-1 & 21,0 & 20,2 & 28,0 & 37,5 \\
\hline
\end{tabular}

'A.D.: alvura de amostras secas ao ar (air-dry), em temperatura de $23 \pm 0,5^{\circ} \mathrm{C}$ e umidade relativa de $50 \pm 1,0 \%$.

${ }^{2}$ O.D.: alvura de amostras secas em estufa (oven-dry), a $103^{\circ} \mathrm{C}$ e $0 \%$ de umidade relativa.

realizada em altas temperaturas e baixo $\mathrm{pH}$, ser capaz de remover praticamente todos os metais de transição, principalmente o ferro, o qual é o principal causador da reversão de alvura (DUARTE; LACHENAL, 2002; EIRAS et al., 2005; GULLICHSEN; SODERHJELM, 1984). Também foi menor para a polpa com estágio PO, em razão da alta estabilidade de alvura do peróxido de hidrogênio presente nesse estágio (COLODETTE et al., 2004; EIRAS et al., 2005; ZERONIAN; INGLESBY, 1995), e esse resultado está de acordo com os investigadores Karim et al. (20l I), trabalho no qual foi verificado que a substituição do estágio $D$ no final da sequência por $\mathrm{PO}$ aumenta a estabilidade de alvura, pois o estágio de peróxido pressurizado no final da sequência de branqueamento, possui o poder de estabilizar os grupos carbonílicos na celulose e hemiceluloses, gerados durante o branqueamento.
$\mathrm{Na}$ Tabela 2, verifica-se que a sequência referência apresentou viscosidade final de 24,2 cP. A sequência $A_{H T} D(E+P) D D$ produziu polpa com a menor viscosidade e, dessa maneira, é esperado que a polpa produzida com o estágio de hidrólise ácida possua maior degradação das fibras e, consequentemente, piores resistências mecânicas. Porém, segundo Almeida (1986), a viscosidade não é um parâmetro confiável para perda de resistência da polpa, somente os testes físicos e mecânicos das fibras, podem indicar se houve verdadeira perda de resistência da fibra.

$O$ processo que utilizou $A_{H T}$ demandou menor quantidade de dióxido de cloro no estágio $D_{0}$ e, consequentemente, um menor custo que a sequência referência, já que o dióxido de cloro apresenta custo superior ao ácido sulfúrico e a hidrólise ácida elimina a necessidade da aplicação de dióxido de cloro nos estágios subsequentes. Deve ser notado que $\circ$ uso do $P_{X}$ elevou significativamente 0 custo total de branqueamento da polpa, sendo $44 \%$ superior ao custo da sequência referência. Esse valor é alto, em razão da grande quantidade de $\mathrm{NaOH}$ aplicada para elevar $\circ \mathrm{pH}$ do estágio $P_{x}$, como também o alto custo do perácido (BRASILEIRO et al., 2002).

\section{Classificação de fibras}

De acordo com a Figura I, a maior quantidade de polpa foi retida na peneira com malha de 50 mesh para todas as sequências de branqueamento. $O$ estágio com peróxido pressurizado e a hidrólise ácida reduziram o tamanho das fibras de celulose fazendo com que aumentasse a quantidade de fibras nas malhas de 100 e 200 mesh do classificador. Uma possível explicação da diminuição do tamanho das fibras é a despolimerização terminal no polímero de celulose no estágio PO, utilizado altas temperaturas e meio alcalino. Para o estágio de hidrolise ácida, ocorreu hidrólise das cadeias celulose em alta temperatura e baixo $\mathrm{pH}$.

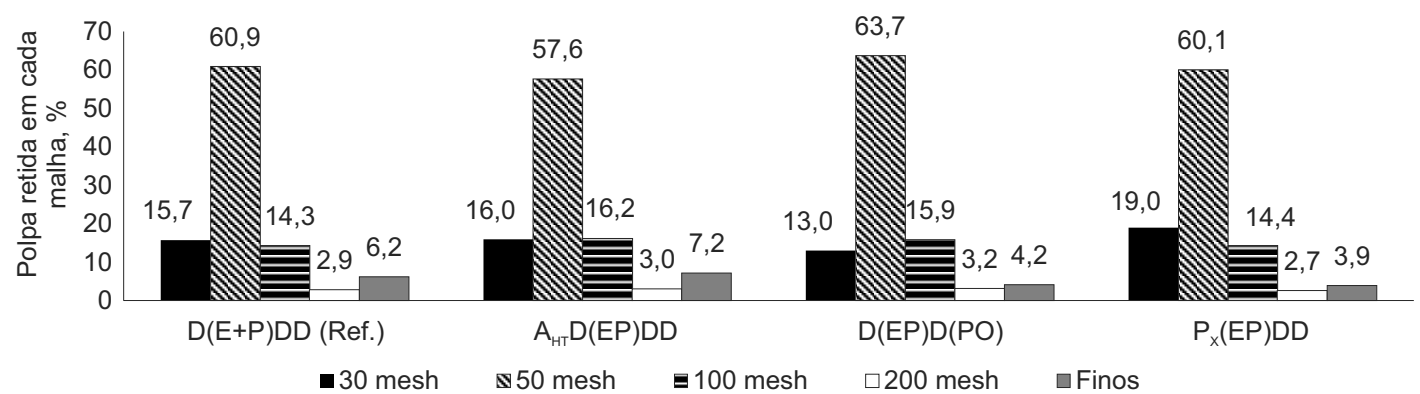

FIGURA I Porcentagem média de polpa retida em cada tanque do classificador.

FIGURE I Average percentage of pulp retained on each classifier chamber. 
De acordo com Silva et al. (1997), a classificação de fibras em Bauer-McNett sofre elevadas variações dependendo das características químicas, físicas e anatômicas da espécie de madeira e do processo de branqueamento utilizado. A quantidade de fibras de tamanhos menores, apresentado pelas sequências com os estágios $\mathrm{A}_{\mathrm{HT}}$ e $\mathrm{PO}$, pode resultar em polpas com menores resistências e viscosidades.

\section{Refino}

$\mathrm{Na}$ Tabela 3, verifica-se o ponto ideal de refino $\left(40^{\circ} \mathrm{SR}\right)$ para cada uma das polpas celulósicas das sequências globais. Era esperado que polpas com menores viscosidades e comprimento de fibras necessitassem menor energia no refino, mesmo considerando a maior remoção de xilanas ocasionados por estágios ácidos. Mas isso não ocorreu, pois a sequência com o estágio $P_{x}$ utilizou uma baixa energia, embora apresente fibras de maior comprimento que a sequência com $\mathrm{A}_{\mathrm{HT}}$ Podese observar que o estágio alcalino no final da sequência promoveu $\circ$ menor requerimento de energia, pelo maior inchamento das fibras de celulose (ANDRADE; COLODETTE, 2010).

Pela Tabela 3, observa-se a influência da ausência dos finos na resistência à drenagem de todas as amostras classificadas em comparação com as amostras globais. Os finos desempenham um papel importante na formação da folha, pois atuam como agentes ligantes das fibras (VOMHOFF; GRUNDSTRÖM, 2003; ZAYTSEVA, 2010). O maior espaço livre existente entre as fibras corresponderam a um menor ${ }^{\circ} \mathrm{SR}$, em razão do baixo teor de finos e, consequentemente, menor resistência à drenabilidade.

A polpa que apresentou maior consumo de energia foi a referência, por apresentar maior viscosidade e estágio ácido no fim da sequência. $O$ menor consumo de energia foi para a polpa com estágio $\mathrm{PO}$, apresentando $70 \%$ a menos de consumo de energia que a referência, em razão do estágio alcalino no final da sequência, no entanto, apresentou também menor viscosidade. Verificou-se que mesmo após o refino, as polpas classificadas apresentaram teor de finos muito abaixo de suas amostras globais.

\section{Testes físicos e mecânicos}

Foram denominadas R Global e R50; A Global e A50; P Global e P50; X Global e X50 as amostras não classificadas (global) e as amostras classificadas e retidas nas malhas de 50 mesh, para as sequências $D(E+P) D D$, $A_{H T} D(E+P) D D, D(E+P) D(P O), P_{X}(E+P) D D$.

Por meio da Figura 2a, observa-se que o índice de tração das amostras R Global, A Global e X Global foram consideradas estatisticamente iguais. A amostra $P$ Global foi a que apresentou menor índice de tração entre as globais, em razão da baixa seletividade do estágio PO em comparação com o estágio de dioxidação da R Global. Era esperado, com base na viscosidade, que a polpa com menor resistência mecânica fosse referente à polpa com estágio de hidrolise ácida, porém esse efeito não foi verificado. Dentre as polpas classificadas, a que a presentou maior índice de tração foi a R50 e A50, sendo estas consideradas estatisticamente iguais às polpas R Global, A Global e X Global, mesmo o teor de finos sendo muito inferior.

Verificou-se diferença significativa do ${ }^{\circ} \mathrm{SR}$ entre as amostras, sendo que a polpa $\times$ Global $\left(40^{\circ} \mathrm{SR}\right)$ apresentou mesmo índice de tração que R50 (27 $\left.{ }^{\circ} \mathrm{SR}\right)$ e A50 ( $\left.24^{\circ} \mathrm{SR}\right)$. Sendo assim, somente a propriedade de resistência à drenagem não deve ser associada exclusivamente ao índice de tração, devendo-se integrar demais fatores com esta propriedade. Esse resultado é referente ao menor teor de finos, coarseness e mesmo comprimento de fibras. A resistência à tração de X50 foi a menor encontrada nessa análise. A amostra $X$ Global, apesar de mesmo índice de tração ao R Global, apresentou para a amostra $\times 50$ índice de tração aproximadamente $48 \%$ inferior à $\times 50$.

TABELA 3 Resistência à drenagem Schopper-Riegler ( $\left.{ }^{\circ} \mathrm{SR}\right)$ das polpas globais e classificadas, refinadas nas mesmas condições que suas amostras globais.

TABLE 3 Global and fractionated pulp drainability ( $\left.{ }^{\circ} \mathrm{SR}\right)$, refined to the same conditions as their global samples.

\begin{tabular}{|c|c|c|c|c|c|c|c|c|c|c|}
\hline \multirow{2}{*}{ Sequência } & \multirow{2}{*}{ Rev. PFI } & \multirow{2}{*}{ PFI (Wh) } & \multicolumn{2}{|c|}{${ }^{\circ} \mathrm{SR}$} & \multicolumn{2}{|c|}{ Comprimento, mm* } & \multicolumn{2}{|c|}{ Finos, \%* } & \multicolumn{2}{|c|}{ Coarseness, g/100m* } \\
\hline & & & Global & Class. & Global & Class. & Global & Class. & Global & Class. \\
\hline $\mathrm{D}(\mathrm{E}+\mathrm{P}) \mathrm{DD}$ (ref.) & 2300 & 60,3 & 40 & 27 & 0,85 & 0,91 & 18,0 & 3,1 & 7,9 & 6,3 \\
\hline$A_{H T} D(E+P) D D$ & 2100 & 50,6 & 40 & 24 & 0,86 & 0,87 & $\mathrm{II}, 4$ & 3,9 & 7,1 & 7,9 \\
\hline $\mathrm{D}(\mathrm{E}+\mathrm{P}) \mathrm{D}(\mathrm{PO})$ & 1500 & 18,0 & 40 & 19 & 0,83 & 0,87 & 10,8 & 3,1 & 7,5 & 8,1 \\
\hline$P_{x}(E+P) D D$ & 1800 & 45,5 & 40 & 21 & 0,83 & 0,87 & 13,3 & 3,4 & 7,4 & 6,0 \\
\hline
\end{tabular}

* Medidos em Fiber Quality Analyser (FQA - LDA02) 
a)

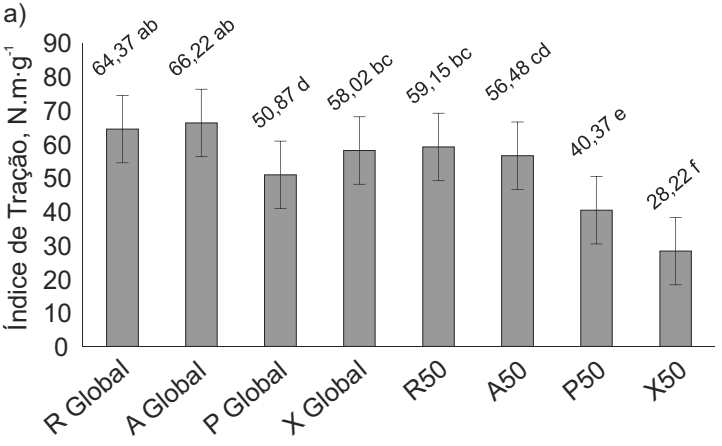

c)

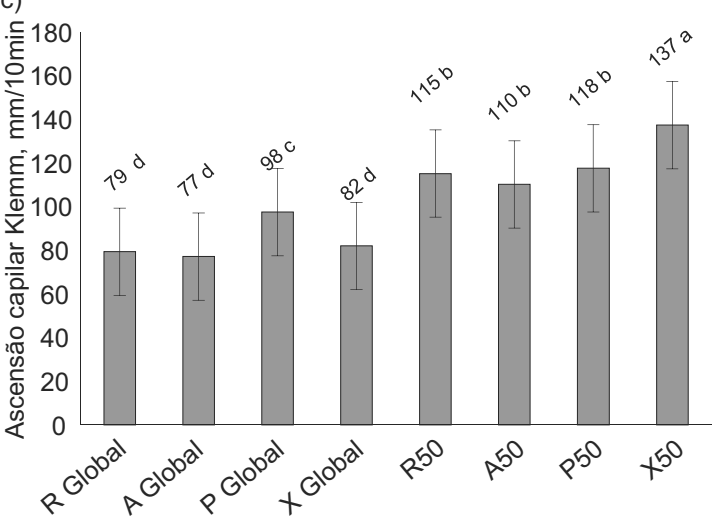

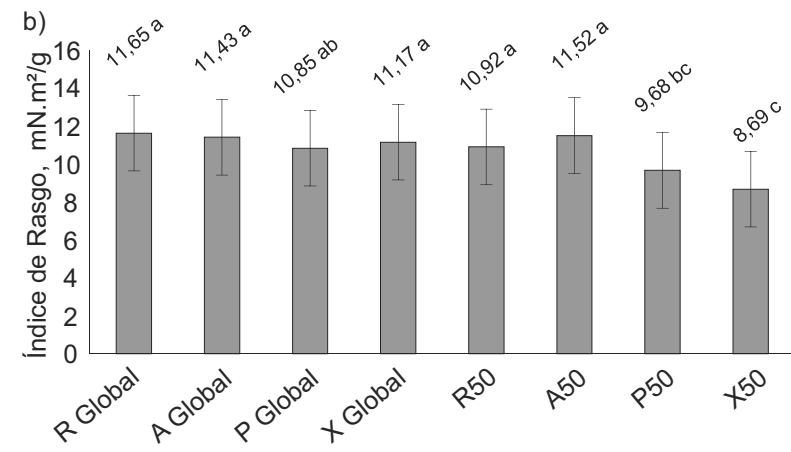

d)

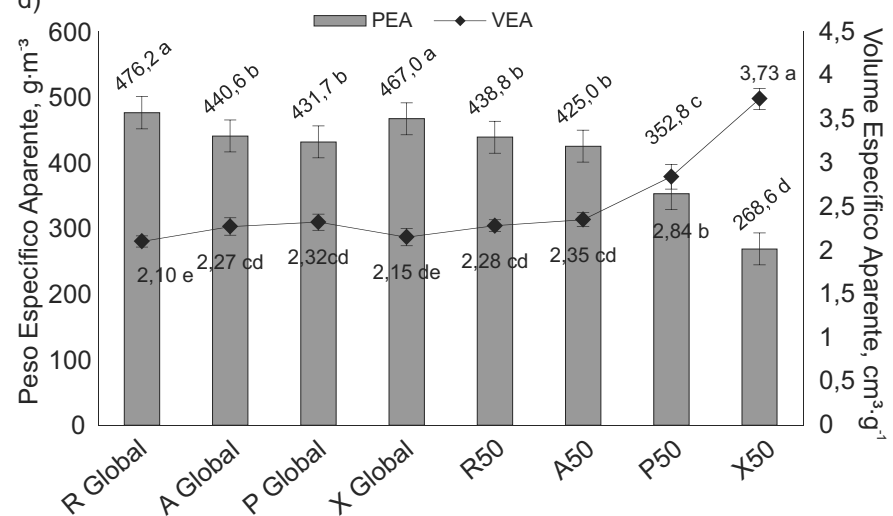

FIGURA 2 a) Índice de tração; b) Índice de rasgo; c) ascensão capilar Klemm; d) peso e volume específico aparente das polpas globais e classificadas. *Médias seguidas por uma mesma letra não apresentam diferença significativa pelo teste de Tukey a $5 \%$ de probabilidade.

FIGURE 2 a) Tensile index; b) tear index; c) Klemm capillary; d) apparent specific weight and apparent specific volume of global and classified pulps. *Means followed by the same letter do not significantly differ by the Tukey test at the $5 \%$ probability level.

Observa-se que as amostras globais não apresentaram diferença entre si para o índice de rasgo, conforme observado na Figura 2b. A amostra P Global foi considerada igual a P50 e esta, diferente das demais. Foi observado redução de $20,4 \%$ da polpa $X 50$ em relação à $\mathrm{R} 50$, devido ao seu menor comprimento de fibras. Apesar de a hidrólise ácida ser mais agressiva aos polissacarídeos, nenhuma diferença de índice de rasgo foi encontrada em relação à polpa R50 após a classificação das fibras, por apresentarem tamanhos de fibras semelhantes. Esse comportamento devese ao fato da resistência ao rasgo estar diretamente relacionada ao comprimento das fibras. Hakanen e Hartler (1995), Page (1994) e Seth e Page (1988), observaram uma relação linear entre índice de rasgo e comprimento médio da fibra. Esse comportamento também foi observado no presente estudo, já que as amostras que apresentaram o mesmo comprimento de fibras, também apresentaram valores de índice de rasgo iguais. O maior índice de rasgo para polpa classificada com maior comprimento de fibras foi também observado no estudo de Carvalho et al. (1997).
$\mathrm{Na}$ Figura 2c, verifica-se que as polpas classificadas apresentaram maior capilaridade em relação às polpas não classificadas. A ausência de finos nas amostras classificadas contribuiu para que apresentassem maior ascensão capilar Klemm, e esse comportamento está de acordo como o estudado por Krogerus e Fagerholm (2002). Como os finos presentes na polpa global ocupam os espaços vazios existentes entre as fibras nas amostras globais, averigua-se a importância dos finos na propriedade de capilaridade.

Dentre as globais, a amostra P Global apresentou - maior valor de capilaridade, pois foi a polpa com o menor número de revoluções no refino. Com o refino, a estrutura do papel torna-se mais consolidada, reduzindo o diâmetro médio aparente dos capilares e lúmen das fibras no papel. Sendo assim, o transporte ascendente de água pela estrutura fibrosa é reduzido (FERREIRA et al., 1997; SANTOS; SANSÍGOLO, 2007).

$\mathrm{Na}$ Figura 2d observa-se que as amostras $\mathrm{R}$ Global e $X$ Global foram consideradas estatisticamente iguais, portanto o peso específicos aparente (PEA) não foi afetado pelo estágio com perácido. As amostras 
A Global e $\mathrm{P}$ Global foram consideradas iguais às amostras R50 e A50. Isso mostra que apesar da menor concentração de finos nessas amostras classificadas, o PEA foi igual às amostras não classificadas com estágio ácido e peróxido pressurizado. De maneira oposta ao PEA, o volume específico aparente (VEA) foi maior para a amostra $X 50$, em razão da refinação ser realizada a menor número de revoluções que a amostra referência. As amostras A Global, P Global, X Global, R50 e A50 formas consideradas estatisticamente iguais. Apesar de as polpas terem sido refinadas ao mesmo número de revoluções, a classificação das fibras afetou o VEA. Esse fato está relacionado ao menor número de ligações entre as fibras, em razão da redução da concentração de finos na polpa (KROGERUS; FAGERHOLM, 2002).

\section{CONCLUSÕES}

Diante das análises do estudo, podem-se inferir as seguintes conclusões: os diferentes estágios de branqueamento geraram polpas celulósicas com comportamento desigual durante a classificação das fibras e testes físicos e mecânicos; foi verificado forte impacto do refino em todas as propriedades das folhas analisadas, para as diferentes classificações; as propriedades de resistência foram afetadas pela seletividade dos reagentes de branqueamento e comprimento de fibras, sendo verificado que para comprimento de fibras maiores, maior resistência das amostras; a resistência à tração não está relacionada com a viscosidade; a sequência com a hidrólise ácida, apesar de menos seletiva, produziu polpas globais e classificadas com as mesmas propriedades da sequência referência; a resistência ao rasgo foi afetada pelo comprimento das fibras e não pelo teor de finos.

\section{AGRADECIMENTOS}

À Fundação de Amparo à Pesquisa do Estado de São Paulo (FAPESP), pelo apoio financeiro recebido.

\section{REFERÊNCIAS}

ALMEIDA, M. L. O. d'. Viscosidade de uma pasta celulósica e a resistência do papel formado. O Papel, São Paulo, v. 46, n. 8, p. 39-42, 1986.

ANDRADE, M. F.; COLODETTE, J. L. Influência do último estágio de branqueamento ECF, na branqueabilidade, no refino e nas propriedades da polpa. Scientia Forestalis, Piracicaba, v. 38, n. 86, p. 135-|46, jun. 2010.
ASSOCIAÇÃO BRASILEIRA DE CELULOSE E PAPEL. Relatório estatístico. São Paulo, 2010. 49 p. Disponível em: <http://www.bracelpa.org.br/bra2/sites/default/files/ estatisticas/rel20l0.pdf>. Acesso em: 27 jan. 2012.

BANAVATH, H. N.; BHARDWAJ, N. K.; RAY, A. K. A comparative study of the effect of refining on charge of various pulps. Bioresource Technology, New York, v. I02, n. 6, p. 4544-455I, 20I I

BRASILEIRO, L. B.; COLODETTE, J. L.; OLIVEIRA, R. C.; VELOSO, D. P. Bleaching of eucalypt kraft pulp with peracids: the effect on pulp characteristics. Appita Journal, Melbourne, v. 55, p. 46I-467, 2002

CARVALHO, K. H. A.; SILVA, M. L.; SOARES, N. S. Competitiveness of Brazilian wood pulp in the international market. Cerne, Lavras, v. I5, n. 4, p. 383-390, 2009.

CARVALHO, M. G.; MARTINS, A. A.; FIGUEREDO, M. M. Fracionamento de pasta kraft de eucalipto: caracterização físico-química e desempenho papeleiro. O Papel, São Paulo, v. 58, n. 7, p. 83-86, 1997.

COLODETTE, J. L.; EIRAS, K. M. M.; OLIVEIRA, R.; VENTORIM, G. Influence of eucalypt wood supply on pulp brightness stability. Appita Journal, Melbourne, v. 57, n. 6, p. $481-487,2004$

CORSON, S. R. Aspect of mechanical pulp fibre separation and development in a disc refiner. Paperi Ja Puu - Paper and Timber, Helsinki, v. 7I, n. 7, p. 80I-8I4, 1989.

DUARTE, A. P.; LACHENAL, D. Hydrogen peroxide production during oxygen bleaching of Eucalyptus globulus kraft pulp: origin of cellulose degradation. Paperi Ja Puu - Paper and Timber, Helsinki, v. 84, n. 4, p. 275-277, 2002.

EIRAS, K. M. M.; COLODETTE, J. L.; CARVALHO, A. M. M. L. Estudo das causas de reversão de alvura de polpas kraft de eucalipto. Cerne, Lavras, v. I I, n. 4, p. 354-368, 2005.

FERREIRA, G. W.; GONZAGA, J. V.; FOELKEL, C. E. B.; ASSIS, T. F.; RATNIEKS, E.; SILVA, M. C. M. Qualidade da celulose kraft-antraquinona de Eucalyptus dunnii plantado em cinco espaçamentos em relação ao Eucalyptus grandis e Eucalyptus saligna. Ciência Florestal, Santa Maria, v. 7, n. I, p. 4I-63, 1997

GIGAC, J.; FIŠEROVÁ, M. Influence of pulp refining on tissue paper properties. TAPPI Journal, Atlanta, v. 7, p. 27-32, Aug. 2008.

GOODING, R. W.; OLSON, J. A. Fractionation in a BauerMcNett classifier. Journal of Pulp and Paper Science, Montreal, v. 27, n. I2, p. 423-428, 200 I.

GULLICHSEN, J.; SODERHJELM, L. On the color reversion of bleached kraft pulp. Paperi ja Puu- Paper and Timber, Helsinki, v. I, p. 34-37, 1984. 
HAKANEN, A.; HARTLER, N. Fiber deformations and strength potential of kraft pulp. Paperi ja Puu- Paper and Timber, Helsinki, v. 77, n. 5, p. 339-34I, 1995.

KARIM, M. R.; ISLAM, M. N.; MALINEN, R. O. Response of Eucalyptus camaldulensis and Acacia mangium kraft pulp in different ECF bleaching options. Wood Science and Technology, Berlin, v. 45, n. 3, p. 473-485, Aug. 201 I.

KROGERUS, B.; FAGERHOLM, K. Fines from different pulps compared by image analysis. Nordic Pulp and Paper Research Journal, Stockholm, v. I7, n. 4, p. 440-444, 2002.

LUNDIN, T.; WURLITZER, F.; PARK, S. W.; FARDIM, P. Análise energética em refinação de baixa consistência de madeira de coníferas. O Papel, São Paulo, v. 70, n. 10, p. 4I-60, 2009.

PAGE, D. H. Note on the mechanism of tearing strength. Tappi Journal, Atlanta, v. 77, n. 3, p. 20I-204, 1994.

SANTOS, S.; SANSÍGOLO, C. A. Influência da densidade básica da madeira de clones de Eucalyptus grandis $x$ Eucalyptus urophylla na qualidade da polpa branqueada. Ciência Florestal, Santa Maria, v. 7, n. I, p. 53-63, jan./mar. 2007.

SETH, R. S. Beating and refining response of some reinforcement pulps. TAPPI Journal, Atlanta, v. 82, n. 3, p. 147-155, 1999.

SETH, R. S.; PAGE, D. H. Fiber properties and tearing resistance. Tappi Journal, Atlanta, v. 7I, n. 2, p. 103-107, 1988.
SILVA, D. J.; OLIVEIRA, R. C.; COLODETTE, J. L.; GOMIDE, J. L. Impacto da qualidade da madeira na deslignificação, no branqueamento e nas propriedades físico-mecânicas da polpa kraft de eucalipto. O Papel, São Paulo, v. 58, n. 2, p. 33-43, 1997.

TAIPALE, T.; ÖSTERBERG, M.; NYKÄNEN, A.; RUOKOLAINEN, J.; LAINE, J. Effect of microfibrillated cellulose and fines on the drainage of kraft pulp suspension and paper strength. Cellulose, London, v. I, n. I7, p. I0051020, 2010.

TECHNICAL ASSOCIATION OF THE PULP AND PAPER INDUSTRY. Standard methods of technical association of the pulp and paper industry. Atlanta: Technical Divisions and Committees, 2002.

VOMHOFF, H.; GRUNDSTRÖM, K. J. Fractionation of a bleached softwood pulp and separate refining of the earlywood- and latewood-enriched fractions. Internationale Papierwirtschaft, Heusenstamm, v. 2, n. I, p. 37-4I, 2003.

ZAYTSEVA, Y. Effect of pulps fractionation on formation and strength properties of laboratory handsheets. 2010. $77 \mathrm{f}$. Dissertation (Master's Degree in Chemical and Process Engineering) - Lappeenranta University of Technology, Lappeenranta, 2010.

ZERONIAN, S. H.; INGLESBY, M. K. Bleaching of cellulose by hydrogen peroxide. Cellulose, London, v. 2, p. 265-272, 1995. 